\title{
Factors associated with myocardial SARS-CoV-2 infection, myocarditis, and cardiac inflammation in patients with COVID-19
}

\author{
Mayara Bearse ${ }^{1}$ - Yin P. Hung $\mathbb{1}^{1,2}$ - Aram J. Krauson $\mathbb{C}^{1} \cdot$ Liana Bonanno $^{1,2} \cdot$ Baris Boyraz $^{1,2}$ - Cynthia K. Harris ${ }^{1,2}$. \\ T. Leif Helland ${ }^{1,2} \cdot$ Caroline F. Hilburn $^{1,2} \cdot$ Bailey Hutchison ${ }^{1,2} \cdot$ Soma Jobbagy ${ }^{1,2} \cdot$ Michael S. Marshall $\mathbb{D}^{1,2}$. \\ Daniel J. Shepherd ${ }^{1,2} \cdot$ Julian A. Villalba ${ }^{1,2} \cdot$ Isabela Delfino $^{3} \cdot$ Javier Mendez-Pena ${ }^{1} \cdot$ Ivan Chebib $^{1,2}$. \\ Christopher Newton-Cheh ${ }^{4,5} \cdot$ James R. Stone $\mathbb{i}^{1,2}$
}

Received: 30 December 2020 / Revised: 12 February 2021 / Accepted: 13 February 2021 / Published online: 17 March 2021

(c) The Author(s), under exclusive licence to United States \& Canadian Academy of Pathology 2021

\begin{abstract}
COVID-19 has been associated with cardiac injury and dysfunction. While both myocardial inflammatory cell infiltration and myocarditis with myocyte injury have been reported in patients with fatal COVID-19, clinical-pathologic correlations remain limited. The objective was to determine the relationships between cardiac pathological changes in patients dying from COVID-19 and cardiac infection by SARS-CoV-2, laboratory measurements, clinical features, and treatments. In a retrospective study, 41 consecutive autopsies of patients with fatal COVID-19 were analyzed for the associations between cardiac inflammation, myocarditis, cardiac infection by SARS-CoV-2, clinical features, laboratory measurements, and treatments. Cardiac infection was assessed by in situ hybridization and NanoString transcriptomic profiling. Cardiac infection by SARS-CoV-2 was present in 30/41 cases: virus ${ }^{+}$with myocarditis $(n=4)$, virus ${ }^{+}$without myocarditis $(n=26)$, and virus ${ }^{-}$without myocarditis $(n=11)$. In the cases with cardiac infection, SARS-CoV-2 $2^{+}$cells in the myocardium were rare, with a median density of 1 cell/ $\mathrm{cm}^{2}$. Virus ${ }^{+}$cases showed higher densities of myocardial $\mathrm{CD} 68^{+}$macrophages and $\mathrm{CD}^{+}$lymphocytes, as well as more electrocardiographic changes $(23 / 27 \mathrm{vs} 4 / 10 ; P=0.01)$. Myocarditis was more prevalent with IL-6 blockade than with nonbiologic immunosuppression, primarily glucocorticoids $(2 / 3$ vs $0 / 14 ; P=0.02)$. Overall, SARS-CoV-2 cardiac infection was less prevalent in patients treated with nonbiologic immunosuppression (7/14 vs 21/24; $P=0.02$ ). Myocardial macrophage and lymphocyte densities overall were positively correlated with the duration of symptoms but not with underlying comorbidities. In summary, cardiac infection with SARS-CoV-2 is common among patients dying from COVID-19 but often with only rare infected cells. Cardiac infection by SARS-CoV-2 is associated with more cardiac inflammation and electrocardiographic changes. Nonbiologic immunosuppression is associated with lower incidences of myocarditis and cardiac infection by SARS-CoV-2.
\end{abstract}

Supplementary information The online version contains supplementary material available at https://doi.org/10.1038/s41379021-00790-1.

James R. Stone

jrstone@ partners.org

1 Department of Pathology, Massachusetts General Hospital, Boston, MA, USA

2 Department of Pathology, Harvard Medical School, Boston, MA, USA

3 Uniube University, Uberaba, Brazil

4 Cardiovascular Research Center \& Center for Genomic Medicine, Massachusetts General Hospital, Boston, MA, USA

5 Program in Medical and Population Genetics, Broad Institute of Harvard and MIT, Boston, MA, USA

\section{Introduction}

While coronavirus disease 2019 (COVID-19), as caused by severe acute respiratory syndrome coronavirus-2 (SARSCoV-2), primarily affects the lungs [1,2], systemic viremia can occur in critically ill patients with COVID-19 [3, 4]. Potential cardiac involvement by SARS-CoV-2 as indicated by dysrhythmias and acute heart failure with reduced ejection fraction has been reported $[5,6]$.

Multiple manifestations of cardiac pathology have been documented in autopsy studies of patients with fatal COVID19, including microvascular thrombi, ischemic injury, right ventricular strain injury, pericarditis, myocardial inflammatory cellular infiltrates, and full myocarditis with myocyte destruction [7]. While studies have detected SARS-CoV-2 in 
the myocardium primarily via RT-PCR on fresh tissue or in situ hybridization (ISH) on formalin-fixed paraffinembedded tissue [8-13], the relationships among cardiac pathological changes, cardiac infection by SARS-CoV-2, and clinical features remain unclear. Clinical trials have demonstrated improved outcomes of hospitalized patients with COVID-19 treated with glucocorticoids [14-17] but not tocilizumab [18-20]. Associations between these medications and myocardial inflammation, myocarditis, and cardiac infection by SARS-CoV-2 have not been reported.

As previous reports have described the cardiac histology in patients dying from COVID-19 [7, 9, 10], the clinical and functional relevance of these findings remains unclear. In this study, we aimed to determine the associations between cardiac pathological changes and the presence of cardiac infection by SARS-CoV-2, clinical features, laboratory measurements, and immunosuppressive treatments.

\section{Subjects and methods}

\section{Patients and study criteria}

The patient cohort included sequential autopsies performed between March and July of 2020 at the Massachusetts General Hospital, Boston, Massachusetts that met the inclusion criteria: a positive antemortem nasopharyngeal swab for SARS-CoV-2, along with clinical and/or postmortem assessment of death due to COVID-19. The study was approved by the Hospital's human subjects institutional review board.

\section{Clinical information}

Medical records were reviewed for baseline patient characteristics, laboratory values, electrocardiography studies (ECGs), cardiac imaging studies, treatments, and durations of illness and hospitalization. For hematologic laboratory values and fibrinogen levels, the lowest recorded value was used. For creatinine and estimated glomerular filtration rate (eGFR), the last value prior to initiation of renal replacement therapy (RRT) was used. For IL-6 levels, the highest level prior to initiation of IL-6 blockade was used. For all other laboratory tests, the highest recorded value was used. Electrocardiographic changes were obtained from the medical records and considered new when not present prior to hospitalization or on previous studies during the terminal hospitalization.

\section{Pathologic analysis}

Postmortem heart weights were compared with the upper limit of normal based on the height and sex [21, 22]. The hearts were examined histologically after formalin fixation and paraffin embedding using 20 blocks of myocardium from the left and right ventricles, along with additional sections of the major coronary arteries and left and right atria. The presence of myocarditis, inflammation, and other patterns of myocardial injury were assessed on hematoxylin and eosin (H\&E)-stained slides. Myocarditis was defined as an inflammatory infiltrate associated with myocyte injury, which was not due to some other cause and was present in multiple foci [7]. The degree of coronary artery disease was estimated based on the percentage of luminal area loss [23]. The degree of inflammation was quantified on the ventricular tissue block with the most inflammatory cells using immunohistochemical stains for CD3, CD4, CD8, and CD68. The cell density positive for each marker was determined by counting the number of cells in ten highpower fields (each $0.23 \mathrm{~mm}^{2}$ ) and was expressed as cells/ $\mathrm{mm}^{2}$. The presence of microthrombi was assessed using H\&E-stained slides and CD61 immunohistochemistry. The presence of right ventricular strain injury was ascertained by C4D immunohistochemistry, which highlights necrotic myocytes. All immunohistochemical stains were performed on routine automated diagnostic staining platforms.

\section{SARS-CoV-2 in situ hybridization}

The presence of SARS-CoV-2 in the heart was assessed by ISH using RNAscope 2.5 LS Probe-V-nCoV2019-S and RNAscope 2.5D DAB on a diagnostic Leica Biosystems BOND-III apparatus. SARS-CoV-2-ISH was obtained on one left ventricle and one right ventricle section for each case utilizing sections with the most inflammation. SARSCoV-2-ISH-stained slides were evaluated by a cardiac pathologist (YPH) blinded to the clinical and pathological data. The total number of SARS-CoV- $2^{+}$cells on the two slides was enumerated. Cells staining positively that were clearly in blood were excluded. Total tissue area on the slides was determined using ImageJ. The density of SARS$\mathrm{CoV}-2^{+}$cells was expressed as cells $/ \mathrm{cm}^{2}$. A case was considered to have SARS-CoV-2 $2^{+}$myocardial cells, if the density of SARS-CoV-2-ISH ${ }^{+}$cells exceeded that of the non-COVID-19 control cases $\left(>0.3\right.$ cells $\left./ \mathrm{cm}^{2}\right)$. The study cases were divided into three groups based on the presence or absence of myocarditis and myocardial SARS-CoV-2 $2^{+}$ cells. The non-COVID-19 control cases for the ISH analysis consisted of 15 autopsies performed at MGH either in 2019 prior to the COVID-19 pandemic $(n=5)$ or in 2020 during the pandemic with a negative SARS-CoV-2 nasopharyngeal swab $(n=10)$, and included nine men and six women with a median age of 67 years (range $45-87$ years). The causes of death for these control cases were malignancy $(n=4)$, aspiration pneumonia $(n=3)$, coronary artery disease $(n=$ $2)$, cirrhosis $(n=2)$, urosepsis $(n=1)$, non-ischemic 
cardiomyopathy $(n=1)$, peritonitis $(n=1)$, and sarcoidosis $(n=1)$.

\section{Transcriptomic profiling}

SARS-CoV-2 viral RNA and ACE2 mRNA were quantified using the NanoString nCounter Coronavirus Panel Plus (Supplementary Table S1) [24]. Normalization of raw data was performed using the nSolver software 4.0. Data were normalized in combination to spiked-in positive controls, spiked-in negative controls, and the five housekeeping genes (ABCF1, G6PD, NRDE2, OAZ1, and PPIA) of SARS-CoV-2-ISH ${ }^{-}$samples as threshold reference.

\section{Composite risk factor scores}

Composite risk factor scores for underlying pathologic and clinical conditions were calculated based on the presence of each of the following: age $>60$ years, BMI $>30 \mathrm{~kg} / \mathrm{m}^{2}$, severe coronary artery disease, heart weight $>$ upper limit of normal, and previous history of diabetes, hypertension, hyperlipidemia, myocardial infarction, autoimmune disease, or smoking. One point was assigned for each condition for a maximum of 10 points.

\section{Statistical analysis}

The primary outcome was the association of cardiac pathological changes with cardiac infection by SARS-CoV2. Secondary outcomes were the associations of cardiac inflammation, myocarditis, and cardiac infection by SARSCoV-2 with clinical features, laboratory measurements, and treatments. Inflammatory cell densities and RNA levels between groups were compared using either $\mathrm{T}$-test or ANOVA with post-test by Bonferroni, after log transformation as appropriate. Other variables were compared between the three groups using $\chi^{2}$ or Kruskal-Wallis with post-test by Wilcoxon. The relationship of inflammation to clinical variables was assessed by Fisher exact test, Wilcoxon test, or linear regression after log transformation as appropriate. All tests were two-sided. $P$ values $<0.05$ were considered significant for hypothesis testing (primary analyses) or hypothesis generating (secondary analyses).

\section{Results}

\section{Pathologically defined patient groups}

Forty-one patients met the study criteria and included 38 inhospital and 3 out-of-hospital deaths (Table 1). There were 27 men and 14 women (median age 67 [range: 21 to $>89$ ] years), including 23 Caucasian, 16 Hispanic, and 2 African American individuals. The 38 hospitalized patients died a median of 17 days after onset of symptoms and 13 days after hospitalization. For each patient, the underlying cause of death was COVID-19; the immediate cause of death was pneumonia/diffuse alveolar damage in 35 patients (Table 2). Based on the pathological presence of myocardial SARS$\mathrm{CoV}-2^{+}$cells by ISH and/or myocarditis, the patients were divided into three groups (Fig. 1): 4 with myocardial SARSCoV- $2^{+}$cells and myocarditis $\left(\mathrm{V}^{+} \mathrm{M}^{+}\right), 26$ with myocardial SARS-CoV-2 ${ }^{+}$cells without myocarditis $\left(\mathrm{V}^{+} \mathrm{M}^{-}\right)$, and 11 without myocardial SARS-CoV-2 $2^{+}$cells or myocarditis $\left(\mathrm{V}^{-}\right.$ $\mathrm{M}^{-}$). No significant differences were detected among the three groups regarding baseline characteristics (including immediate cause of death, postmortem interval, heart weight, frequency of severe coronary artery disease, or composite cardiac risk factor score), except that nonCaucasian race was more prevalent in patients without myocardial SARS-CoV-2 ${ }^{+}$cells (Tables 1 and 2).

Quantification of cardiac RNA levels by NanoString in 23 cases, all with postmortem intervals less than $48 \mathrm{~h}$, showed that the SARS-CoV-2-ISH ${ }^{+}$cases had higher levels of SARS-CoV-2 antisense RNA indicating viral replication in the heart, and had lower levels of the SARS-CoV-2 receptor ACE2 (Fig. 2). One of the $\mathrm{V}^{-} \mathrm{M}^{-}$cases, which was negative for SARS-CoV-2 by ISH, showed relatively high levels of positive-strand SARS-CoV-2 sequences by NanoString, but not the antisense SARS-CoV-2 RNA strand sequence, likely representing non-replicating packaged virions in the blood.

\section{Variations in cardiac inflammation across the patient groups}

In three of the four patients with myocarditis, the histology in the ventricles demonstrated lymphocytic myocarditis with multiple small discrete foci of lymphocyte predominant inflammation associated with myocyte injury (Fig. 1). The fourth patient had a more extensive granulomatous myocarditis with eosinophils and giant cells (Fig. 3); this patient had been treated with tocilizumab. In three of the four patients with myocarditis, the lymphocytes were CD4 predominant, and in the fourth patient the lymphocytes were CD8 predominant. Compared with the 11 virus-negative cases, the 30 cases with SARS-CoV-2 ${ }^{+}$ myocardial cells showed increased densities of myocardial $\mathrm{CD} 8^{+}$macrophages, $\mathrm{CD}^{+}$T-lymphocytes, and $\mathrm{CD} 4^{+}$ helper T-lymphocytes, but not $\mathrm{CD}^{+}$cytotoxic $\mathrm{T}$ lymphocytes (Figs. 1 and 4 and Supplementary Fig. S1). Compared with both the $\mathrm{V}^{+} \mathrm{M}^{-}$and $\mathrm{V}^{-} \mathrm{M}^{-}$groups, the four patients with myocarditis showed increased densities of myocardial $\mathrm{CD} 68^{+}$macrophages, $\mathrm{CD}^{+}$T-lymphocytes, 
Table 1 Baseline patient characteristics.

\begin{tabular}{|c|c|c|c|c|}
\hline & Virus $^{+}$with myocarditis & Virus $^{+}$without myocarditis & Virus $^{-}$without myocarditis & $P^{\mathrm{a}}$ \\
\hline$n$ & 4 & 26 & 11 & \\
\hline Age (years) & $60(55-64)$ & $71(33->89)$ & $62(21->89)$ & 0.40 \\
\hline Male sex & $2(50)$ & $19(73)$ & $6(55)$ & 0.43 \\
\hline $\operatorname{BMI}\left(\mathrm{kg} / \mathrm{m}^{2}\right)$ & $33(29-36)$ & $30(20-70)$ & $32(22-60)$ & 0.36 \\
\hline Caucasian ethnicity & $4(100)$ & $16(62)$ & $3(27)$ & 0.03 \\
\hline Hypertension & $2(50)$ & 18 (69) & $7(64)$ & 0.74 \\
\hline Diabetes mellitus & $2(50)$ & $9(35)$ & $3(27)$ & 0.71 \\
\hline Hyperlipidemia & $1(25)$ & $13(50)$ & $3(27)$ & 0.34 \\
\hline History of CAD & $0(0)$ & $2(8)$ & $0(0)$ & 0.55 \\
\hline History of autoimmune disease & $0(0)$ & $3(12)$ & $1(9)$ & 0.77 \\
\hline Statin & $1(25)$ & $12(48), n=25$ & $3(27)$ & 0.41 \\
\hline ACE inhibitor & $1(25)$ & $2(8), n=25$ & $2(18)$ & 0.51 \\
\hline Calcium channel blocker & $0(0)$ & $4(16), n=25$ & $1(9)$ & 0.62 \\
\hline Beta-blocker & $1(25)$ & $9(36), n=25$ & $4(36)$ & 0.91 \\
\hline Aspirin & $0(0)$ & $10(40), n=25$ & $2(18)$ & 0.16 \\
\hline History of smoking (current or former) & $2(67), n=3$ & $13(50)$ & $4(40), n=10$ & 0.31 \\
\hline Baseline creatinine (mg/dL) & $1.1, n=1$ & $1.0(0.6-1.6), n=19$ & $1.1(0.8-2.9), n=5$ & 0.74 \\
\hline Baseline BUN (mg/dL) & $19, n=1$ & $17(11-62), n=17$ & $19(8-27), n=5$ & 0.88 \\
\hline
\end{tabular}

Data are expressed as median (range) or $n(\%)$.

For each characteristic, the number of patients is indicated when information regarding the characteristic was not available for all of the patients in the group.

$A C E$ angiotensin-converting enzyme, $B M I$ body mass index, $B U N$ blood urea nitrogen, $C A D$ coronary artery disease.

${ }^{\mathrm{a}} \chi^{2}$ or Kruskal-Wallis.

and $\mathrm{CD} 4^{+}$helper T-lymphocytes, but not $\mathrm{CD} 8^{+}$cytotoxic T-lymphocytes. Similar inflammatory infiltrates were seen within the atrial tissue, with one of the $\mathrm{V}^{+} \mathrm{M}^{+}$cases showing involvement of the atria by myocarditis (Supplementary Fig. S2).

\section{Characteristics of cardiac SARS-CoV-2-ISH ${ }^{+}$cells}

The SARS-CoV-2 ${ }^{+}$cells consisted predominantly of cells in the interstitium, consistent with macrophages, showing either focal dot-like cytoplasmic staining or diffuse cytoplasmic staining (Fig. 1). There were also rare cells with the histologic localization and features of endothelial cells, which also showed either focal dot-like cytoplasmic staining or diffuse cytoplasmic staining (Supplementary Fig. S3). In addition, there were rare apparent myocytes with focal cytoplasmic dot-like staining for SARS-CoV-2 (Supplementary Fig. S3). CD61 ${ }^{+}$microthrombi were present in $3 / 8(38 \%)$ patients with apparent SARS-CoV-2 ${ }^{+}$endothelial cells compared with $2 / 33(6 \%)$ patients without apparent SARS-CoV-2 ${ }^{+}$endothelial cells $(P=0.04)$. Myocarditis was present in $3 / 6(50 \%)$ patients with apparent SARSCoV-2 ${ }^{+}$myocytes, compared with $1 / 35$ (3\%) patients without apparent SARS-CoV-2 ${ }^{+}$myocytes $(P=0.007)$.

\section{Clinical and laboratory features of the pathologically defined patient groups}

There were non-significant trends toward increasing durations of illness and hospitalization for the $\mathrm{V}^{+} \mathrm{M}^{+}$patients compared with the $\mathrm{V}^{+} \mathrm{M}^{-}$patients, and also for the $\mathrm{V}^{+} \mathrm{M}^{-}$ patients compared with the $\mathrm{V}^{-} \mathrm{M}^{-}$patients (Fig. 5 and Supplementary Table S2). Serum levels of C-reactive protein and erythrocyte sedimentation rate did not differ significantly among the three groups. Compared with the $\mathrm{V}^{+} \mathrm{M}^{-}$group, both the $\mathrm{V}^{+} \mathrm{M}^{+}$and $\mathrm{V}^{-} \mathrm{M}^{-}$groups showed more severe indices of multisystem disease, including higher creatinine, lower eGFR, and higher AST/ALT. The $\mathrm{V}^{-} \mathrm{M}^{-}$group showed higher levels of D-dimer and $\mathrm{LDH}$ than the $\mathrm{V}^{+} \mathrm{M}^{-}$group. Among the 38 in-patient deaths, those with myocarditis were more likely to have received RRT, compared with those without myocarditis (4/4 vs 6/ 34; $P=0.002$ ).

\section{Immunomodulatory and antiviral therapy}

For the 38 in-hospital deaths, 19 patients did not receive immunosuppression. Fourteen patients received nonbiologic immunosuppression: hydrocortisone $(n=8)$, 
Table 2 Autopsy findings.

\begin{tabular}{|c|c|c|c|c|}
\hline & Virus $^{+}$with myocarditis & Virus $^{+}$without myocarditis & Virus $^{-}$without myocarditis & $P^{\mathrm{a}}$ \\
\hline$n$ & 4 & 26 & 11 & \\
\hline SARS-CoV-2 ${ }^{+}\left(\right.$cells $\left./ \mathrm{cm}^{2}\right)$ & $1.2(0.5-3.0)$ & $1.2(0.4-7.4)$ & $0.2(0.0-0.3)$ & $<0.001$ \\
\hline $\mathrm{CD} 61^{+}$microthrombi present & $1(25)$ & $4(15)$ & $0(0)$ & 0.30 \\
\hline Right ventricular strain injury & $0(0)$ & $3(12)$ & $2(18)$ & 0.63 \\
\hline Focal isolated pericarditis & $2(50)$ & $6(23)$ & $1(9)$ & 0.23 \\
\hline Postmortem interval (h) & $20(16-26)$ & $20(4-190)$ & $26(9-96)$ & 0.82 \\
\hline Heart weight $(\mathrm{g})$ & $593(480-627), n=3$ & 449 (204-646) & $480(267-738)$ & 0.24 \\
\hline Heart weight/upper limit of normal & $1.25(1.04-1.53), n=3$ & $1.02(0.48-1.67)$ & $1.18(0.72-1.86)$ & 0.26 \\
\hline Severe coronary artery disease & $1(25)$ & $5(19)$ & $3(27)$ & 0.85 \\
\hline Transthyretin amyloidosis & $0(0)$ & $3(12)$ & $0(0)$ & 0.39 \\
\hline Composite risk factor score & $4.0(2-6)$ & $4.5(0-6)$ & $4.0(1-6)$ & 0.93 \\
\hline $\begin{array}{l}\text { Immediate cause of death due to pneumonia/diffuse } \\
\text { alveolar damage }\end{array}$ & $3(75)$ & $23(88)$ & $9(82)$ & 0.72 \\
\hline Other immediate causes of death & RP bleed, $n=1$ & $\begin{array}{l}\text { Refractory VT, } n=1 \\
\text { Acute HF, } n=1 \\
\text { PE, } n=1\end{array}$ & $\begin{array}{l}\text { Abdominal organ } \\
\text { ischemia, } n=2\end{array}$ & \\
\hline
\end{tabular}

Data are expressed as median (range) or $n(\%)$.

For each characteristic, the number of patients is indicated when information regarding the characteristic was not available for all of the patients in the group.

$H F$ heart failure, $P E$ pulmonary embolism, $R P$ retroperitoneal, $V T$ ventricular tachycardia.

${ }^{\mathrm{a}} \chi^{2}$ or Kruskal-Wallis.

methylprednisolone $(n=2)$, hydrocortisone with tacrolimus $(n=1)$, dexamethasone $(n=1)$, sulfasalazine $(n=1)$, and $\operatorname{MMF}(n=1)$. Sulfasalazine and tacrolimus/MMF were prehospitalization treatments for ulcerative colitis $(n=1)$ and renal transplantation $(n=2)$, respectively, which were continued during terminal hospitalization. One of the patients who received nonbiologic immunosuppression also received IL-6 blockade, and one was randomized to IL-6 blockade or placebo. Three patients received IL-6 blockade with tocilizumab for more than 3 weeks (23-39 days) prior to death without nonbiologic immunosuppression, and two were randomized to tocilizumab or placebo without nonbiologic immunosuppression.

There were non-significant trends toward increased frequency of treatment with nonbiologic immunosuppression in the $\mathrm{V}^{-} \mathrm{M}^{-}$group compared with the other two groups, and there were non-significant trends toward higher frequency of IL-6 blockade with tocilizumab in the $\mathrm{V}^{+} \mathrm{M}^{+}$group compared with the other two groups (Fig. 5). Myocarditis was more frequent in patients known to have received IL-6 blockade without nonbiologic immunosuppression than in patients who received nonbiologic immunosuppression $(2 / 3$ vs $0 / 14, P=0.02$ ). Myocarditis was not present in one patient who received tocilizumab and dexamethasone starting 7 days prior to death or in three patients who were randomized to IL- 6 blockade or placebo (tocilizumab $n=2$, sarilumab $n=1$ ) $7-10$ days prior to death, one of whom also received hydrocortisone. Overall, cardiac infection by SARS-CoV-2 was less prevalent in patients treated with nonbiologic immunosuppression, compared to those without $(7 / 14$ vs $21 / 24 ; P=0.02)$.

\section{Anticoagulation}

Of 38 in-patient deaths, 33 patients received anticoagulation: heparin (prophylactic only $n=13$, infusion $n=$ 13), enoxaparin ( $n=19,30-100 \mathrm{mg}$ daily), warfarin $(n=$ $2)$, apixaban $(n=1)$. Of the five patients who had microthrombi, four were in-patient deaths of whom two received anticoagulation with heparin infusions. There was a nonsignificant trend toward the association of cardiac microthrombi with the absence of anticoagulation among the inpatient deaths $(2 / 5$ vs $2 / 33, P=0.08)$.

\section{Serum biomarker, electrocardiographic, and echocardiographic assessments}

Of 36 patients with high-sensitivity troponin $\mathrm{T}$ measurements, 35 (97\%) had elevated values (>14 ng/L), which did not significantly differ among the three groups (Supplementary Table S2). Of 30 patients with NT-pro-BNP measurements, 20 (67\%) had elevated values, with no significant differences among the three groups. Cardiac rhythm assessment by ECG and/or rhythm strip were 

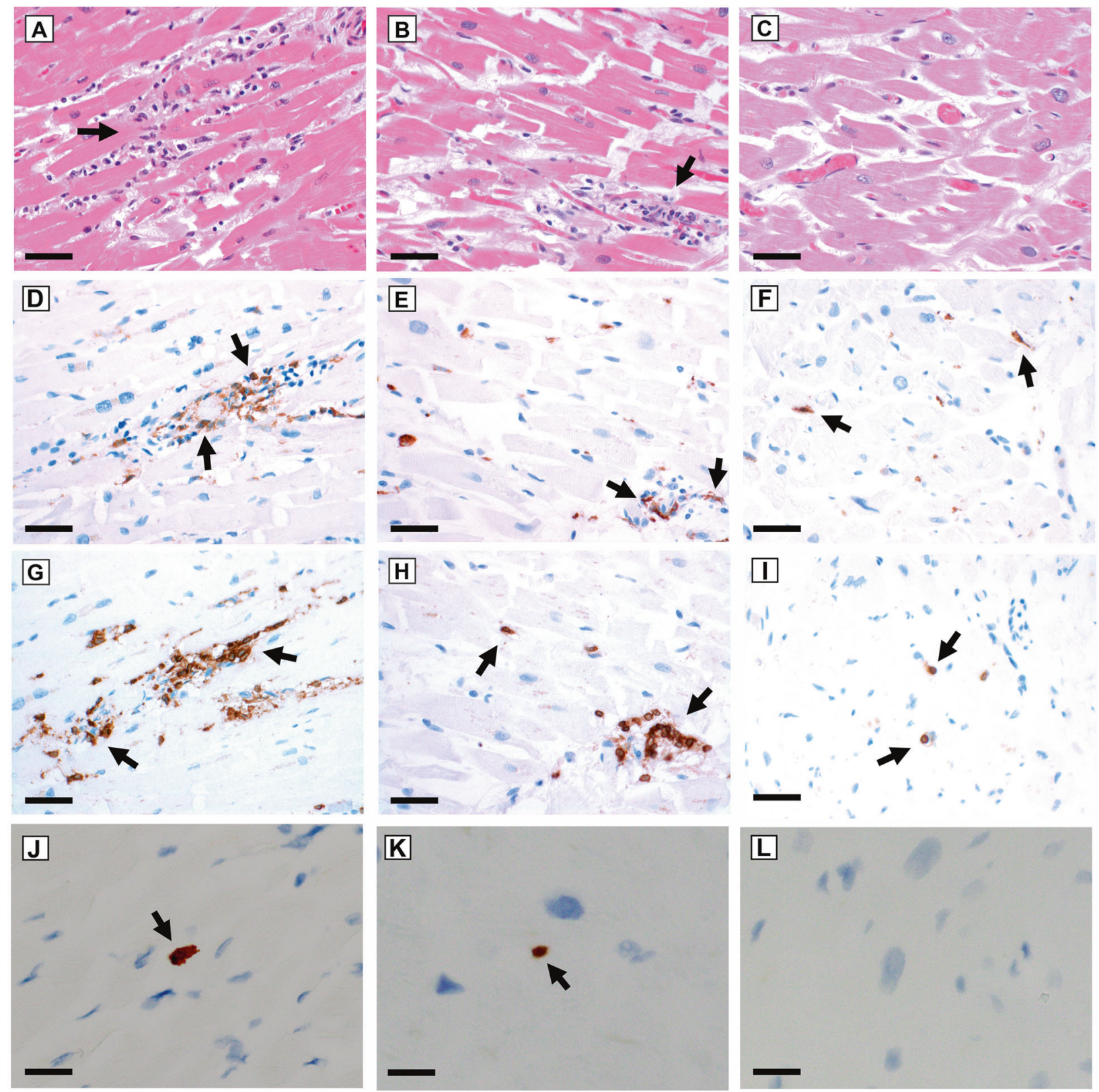

Fig. 1 Histology of the three pathologically defined patient groups. Depicted are histologic images of slides stained with H\&E (A-C), immunohistochemistry for the macrophages marker CD68 (D-F), immunohistochemistry for the T-lymphocyte marker CD3 (G-I), and in situ hybridization for SARS-CoV-2 (J-L) for cases with myocarditis along with SARS-CoV-2 $2^{+}$cells in the myocardium $\left(\mathrm{V}^{+} \mathrm{M}^{+}, \mathbf{A}\right.$, $\mathbf{D}, \mathbf{G}, \mathbf{J})$, negative for myocarditis but with SARS-CoV- $2^{+}$cells in the

available for 37 of 38 in-patient deaths. Three patients had a prior history of atrial fibrillation. In the remaining 34 patients, new atrial fibrillation or premature atrial complexes occurred more frequently in patients with cardiac SARS-CoV-2 infection than those without (11/24 vs 0/10; $P=0.01)$. Additional new electrocardiographic changes included QTc prolongation $(n=8)$, nonspecific ST segment myocardium $\left(\mathrm{V}^{+} \mathrm{M}^{-}, \mathbf{B}, \mathbf{E}, \mathbf{H}, \mathbf{K}\right)$, and negative for both myocarditis and myocardial SARS-CoV-2 $2^{+}$cells $\left(\mathrm{V}^{-} \mathrm{M}^{-}, \mathbf{C}, \mathbf{F}, \mathbf{I}, \mathbf{L}\right)$. There is inflammation with myocardial injury (arrow) in the $\mathrm{V}^{+} \mathrm{M}^{+}$case (A) compared with inflammation (arrow) without injury in the $\mathrm{V}^{+} \mathrm{M}^{-}$case (B). Arrows indicate $\mathrm{CD} 68^{+}$macrophages $(\mathbf{D}-\mathbf{F}), \mathrm{CD}^{+}$lymphocytes (G-I), and SARS-CoV-2 ${ }^{+}$cells $(\mathbf{J}, \mathbf{K})$. Scale bars represent $40 \mu \mathrm{m}(\mathbf{A}-$ I) and $80 \mu \mathrm{m}(\mathbf{J}-\mathbf{L})$.

and/or $\mathrm{T}$ wave abnormalities $(n=7)$, atrioventricular or bundle branch blocks $(n=3)$, and intraventricular conduction defects $(n=3)$. Patients with cardiac SARS-CoV-2 infection were significantly more likely to show electrocardiographic changes $(23 / 27$ vs $4 / 10 ; P=0.01)$.

Limited point of care or full echocardiography was performed in 16 patients (median 8 [range 0.5-27] days prior 


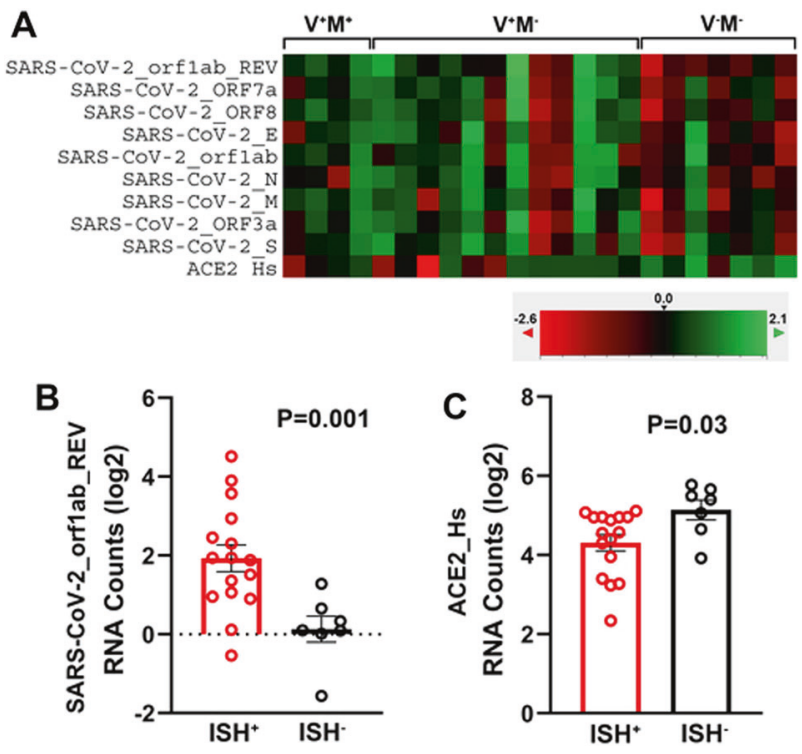

Fig. 2 Transcriptomic assessment of SARS-CoV-2 RNA levels and ACE2 mRNA levels. A heatmap of RNA levels (A) shows trends toward increased SARS-CoV-2-positive-strand RNA transcripts in the SARS-CoV-2-ISH ${ }^{+}$cases $(P=0.04-0.29)$. The color guide shows the corresponding Z-scores for the RNA levels. One $\mathrm{V}^{-} \mathrm{M}^{-}$case had relatively strong expression of the eight positive-strand sequences but not the antisense sequence (SARS-CoV-2_orf1ab_REV) likely due to non-replicating virions in the blood. In the SARS-CoV-2-ISH ${ }^{+}$cases, there was significantly more SARS-CoV-2 antisense RNA, indicating viral replication in the heart $(\mathbf{B})$, as well as downregulation of the SARS-CoV-2 receptor ACE2 (C). Bars indicate mean \pm SE.

to death) and was more frequently obtained in patients subsequently found to have pathologic myocarditis (4/4 vs $12 / 34, P=0.02)$. All four myocarditis patients had normal left ventricular function on the echocardiography (performed within 1 week of death for three patients). Reduced left ventricular ejection fraction (ranging from 20 to 50\%) was noted in $\mathrm{V}^{+} \mathrm{M}^{-}$patients but not in $\mathrm{V}^{-} \mathrm{M}^{-}$patients (3/7 vs $0 / 5, P=0.20$ ). Left ventricular wall abnormalities were noted in patients with or without cardiac SARS-CoV-2 infection $(4 / 11$ vs $1 / 5 ; P=1.00)$.

\section{Clinical features correlating with myocardial inflammation}

Analysis of the associations of clinical and laboratory features with the degree of myocardial inflammation, irrespective of pathologically defined patient groups, is shown in Fig. 6 and Supplementary Tables S3 and S4. There were no significant associations of the inflammatory cell densities with baseline patient characteristics or composite cardiac risk factor scores. The densities of myocardial $\mathrm{CD} 68^{+}$ macrophages, $\mathrm{CD}^{+}$lymphocytes, $\mathrm{CD} 4^{+}$helper lymphocytes, and $\mathrm{CD}^{+}$cytotoxic lymphocytes each positively correlated with the duration of symptoms, and each negatively correlated with the red blood cell concentrations. All

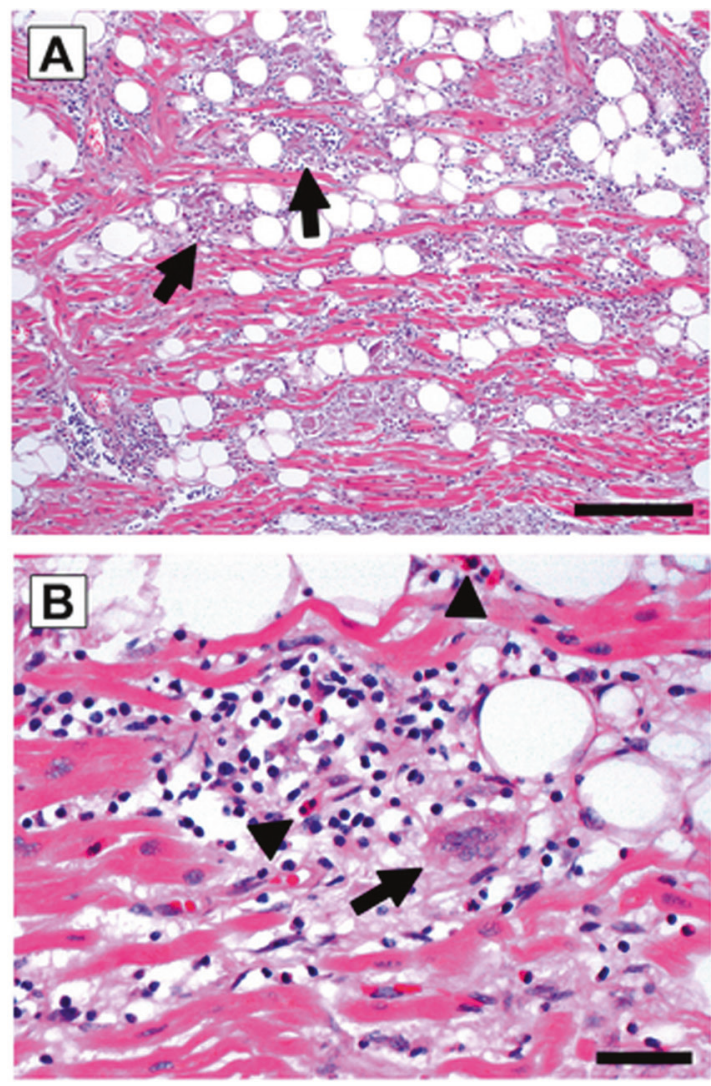

Fig. 3 Myocarditis with eosinophils and giant cells in a patient who died from COVID-19. Depicted are H\&E-stained sections. At low power (A), there is diffuse infiltration of the myocardium by inflammation (arrows). At higher magnification (B), both eosinophils (arrow heads) and giant cells (arrow) are identified in the inflammatory infiltrate). Scale bars represent $200 \mu \mathrm{m}$ (A) and $40 \mu \mathrm{m}$ (B).

four inflammatory cell types showed non-significant positive correlative trends with level of serum IL-6, when measured $(n=16)$.

Compared with those treated only with IL-6 blockade $(n=3), 14$ patients treated with nonbiologic immunosuppression had significantly lower densities of myocardial $\mathrm{CD} 8^{+}$macrophages, $\mathrm{CD}^{+}$T-lymphocytes, and $\mathrm{CD}^{+}$ helper T-lymphocytes (Fig. 6). The patients treated with nonbiologic immunosuppression had fewer myocardial $\mathrm{CD}^{+}{ }^{+}$helper T-lymphocytes than the 19 patients without immunomodulatory therapy. Patients treated with only IL-6 blockade had increased levels of myocardial $\mathrm{CD}^{+} 8^{+}$macrophages compared with patients with no immunosuppression.

\section{Discussion}

This study identified an important association between cardiac SARS-CoV-2 infection and increased densities of myocardial macrophages and lymphocytes. Our observations on SARS-CoV-2 are reminiscent of a prior study on 

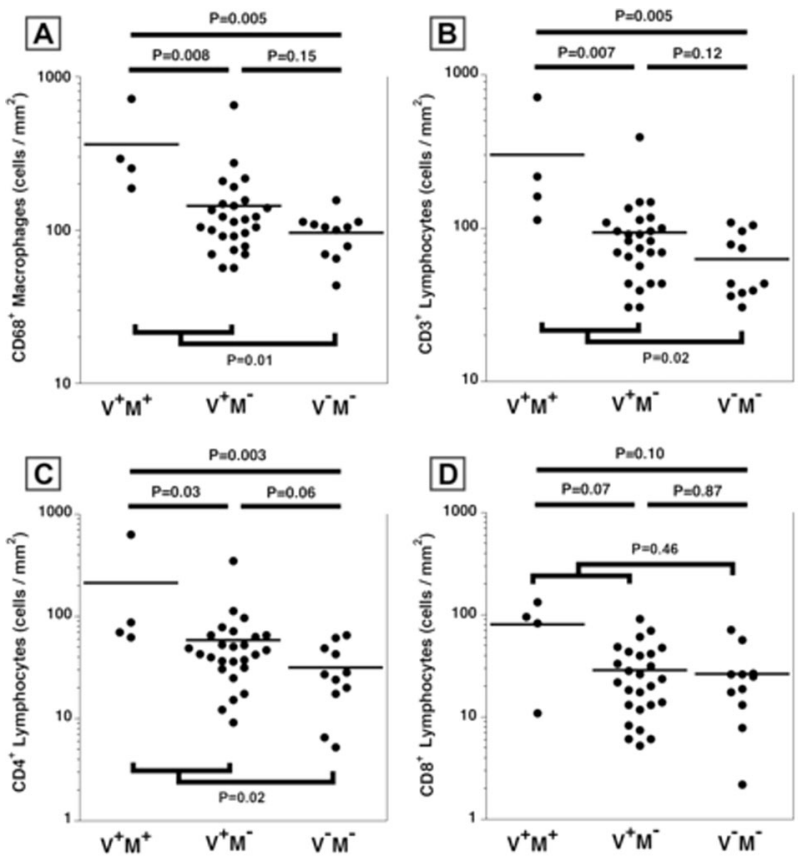

Fig. 4 Associations between myocardial inflammatory cell densities and the presence of myocarditis and cardiac infection by SARS-CoV-2. Depicted are dot plots comparing the densities of $\mathrm{CD} 8^{+}$macrophages $(\mathbf{A}), \mathrm{CD}^{+}{ }^{\mathrm{T}}$-lymphocytes $(\mathbf{B}), \mathrm{CD} 4^{+}$helper $\mathrm{T}$ lymphocytes $(\mathbf{C})$, and $\mathrm{CD}^{+}$cytotoxic T-lymphocytes $(\mathbf{D})$ between the three pathologically defined patient groups $\left(\mathrm{V}^{+} \mathrm{M}^{+}, \mathrm{V}^{+} \mathrm{M}^{-}\right.$, and $\left.\mathrm{V}^{-} \mathrm{M}^{-}\right)$ as well as the patients with and without cardiac infection by SARS$\mathrm{CoV}-2$ overall. For each cell marker the three groups were first compared using Kruskal-Wallis $[P=0.006(\mathbf{A}), P=0.005$ (B), $P=0.008(\mathbf{C}), P=0.17(\mathbf{D})]$ and then the individual groups were compared using Wilcoxon test.

SARS, in which the presence of SARS-CoV in myocardial tissue was associated with increased myocardial macrophages [25]. In patients with SARS-CoV-2 cardiac infection, infected cells were rare (median $1 \mathrm{cell} / \mathrm{cm}^{2}$ ). As electron microscopy analyses typically utilize small pieces of myocardium $\left(\sim 1 \mathrm{~mm}^{2}\right)$, it would be expected to take 100 such analyses to identify one virus-infected cell in the myocardium in these patients.

A small fraction of the patients with cardiac SARS-CoV2 infection met criteria for myocarditis. In three of the four myocarditis patients, the myocarditis was classified as lymphocytic myocarditis. In the remaining patient, the myocarditis was granulomatous with giant cells and eosinophils, with the degree of injury exceeding that typically found in hypersensitivity reactions. While the granulomatous characteristics of the myocarditis may be secondary to the IL-6 blockade this patient had received, this may represent an unusual manifestation of SARS-CoV-2 cardiac infection, given that other causes of myocarditis such as immune checkpoint inhibition can result in both lymphocytic myocarditis and granulomatous myocarditis with eosinophils and giant cells [26].
The extent of myocarditis was relatively mild in three patients, being limited to multiple small discrete foci, but was more extensive in the fourth patient. Functionally, the four patients with myocarditis showed preserved left ventricular ejection fraction on echocardiography obtained 4-21 days prior to death, and in none of these patients was the myocarditis considered the cause of death. However, all of the myocarditis patients required RRT, which may indicate hemodynamic instability in this group of patients. SARS-CoV-2 cardiac infection was associated with increased cardiac rhythm changes, particularly atrial fibrillation and premature atrial complexes. Atrial fibrillation has been identified as a predictor of poor outcome in patients with COVID-19 [30, 31]. Notably, the two patients with cardiac-related immediate causes of death (acute heart failure and refractory ventricular tachycardia) and the three patients with reduced left ventricular ejection fraction all harbored SARS-CoV-2 $2^{+}$cells in their myocardium.

Unexpected secondary observations from this study included the associations between apparent SARS-CoV- $2^{+}$ myocytes and the presence of myocarditis, and between apparent SARS-CoV-2 ${ }^{+}$endothelial cells and the presence of cardiac microthrombi. SARS-CoV-2 has been shown to be able to infect human cardiac myocytes in vitro [27], and such cardiac myocyte infection could result in the pathology of myocarditis. However, only rare apparent SARS-CoV-2 $2^{+}$ myocytes were identified in this study, suggesting cardiac myocytes may be relatively resistant to infection by this virus. Cardiac myocyte infection by SARS-CoV-2 in vitro is associated with decreased ACE2 mRNA levels [27], as was observed with the patients with cardiac SARS-CoV-2 infection here. The association of cardiac microthrombi with apparent SARS-CoV- $2^{+}$endothelial cells may provide potential mechanistic insights into why some patients with COVID-19 develop thrombi despite anticoagulation [28, 29]. Despite these intriguing associations, definitive identification of the cardiac cell types infected by SARS-CoV-2, and the alterations in ACE2 expression accompanying the infection of these specific cells will require additional studies.

Comorbidities are present in many patients with COVID19 with critical illness. However, cardiac inflammation was associated with neither the underlying medical conditions nor the composite risk factor scores. Irrespective of the presence of myocarditis or cardiac SARS-CoV-2 infection, myocardial inflammation in these patients correlated positively and strongly with the duration of symptoms and hospitalization and negatively with the red blood cell count, suggesting that myocardial inflammatory infiltrates may result in many patients with a long COVID-19 illness prior to death. There were non-significant trends between myocardial inflammatory cells and serum IL-6 levels, suggesting systemically elevated cytokines could be promoting myocardial inflammation. 
Fig. 5 Associations of clinical and laboratory features with the pathologically defined patient groups. Depicted are dot plots (A, B, D, E, G, H, J, $\mathbf{K})$ and bar graphs $(\mathbf{C}, \mathbf{F}, \mathbf{I}, \mathbf{L})$ comparing selected clinical and laboratory features between the three pathologically defined patient groups. For each cell marker the three groups were first compared using

Kruskal-Wallis $[P=0.06(\mathbf{A})$, $P=0.12(\mathbf{B}), P=0.002(\mathbf{D})$, $P=0.004(\mathbf{E}), P=0.03(\mathbf{G})$, $P=0.006(\mathbf{H}), P=0.01(\mathbf{J})$, $P=0.03(\mathbf{K})]$ or $\chi^{2}[P=0.02$ (C), $P=0.0003(\mathbf{F}), P=0.02$ (I), $P=0.03(\mathbf{L})]$, and then the individual groups were compared using either Wilcoxon test or Fisher test.
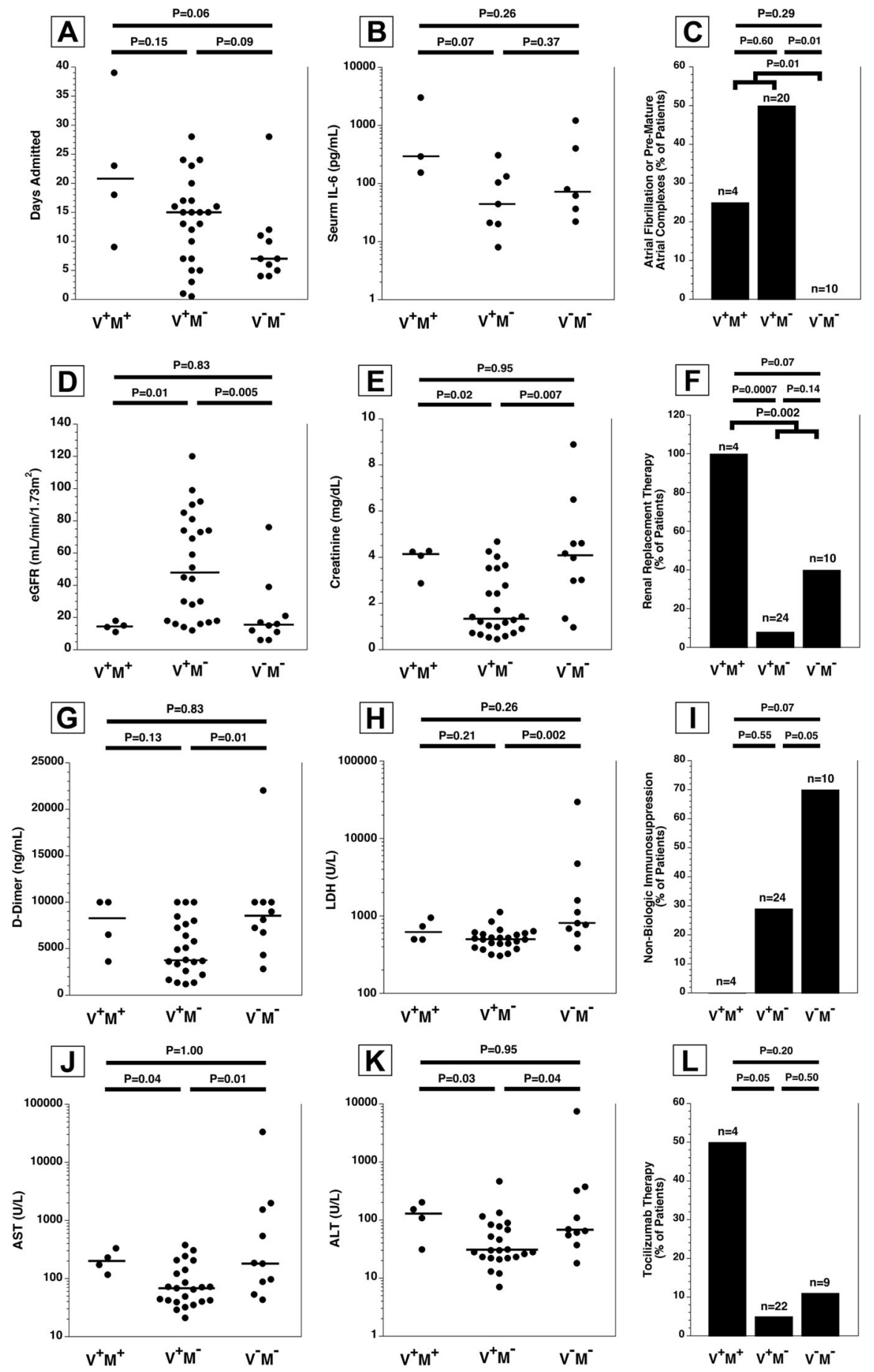

An interesting observation in this study was that treatment with nonbiologic immunosuppression, predominantly glucocorticoids, was associated with a lower prevalence of myocarditis and less myocardial inflammation compared with treatment with IL-6 blockade. Nonbiologic immunosuppression was also associated with a lower rate of cardiac infection by SARS-CoV-2 compared with patients not receiving nonbiologic immunosuppression. In this retrospective study, it cannot be excluded that patients who appeared more "inflamed" by biomarkers were preferentially treated with IL-6 blockade rather than glucocorticoids. Nonetheless, our observations are in agreement with outcomes from clinical trials. In recent randomized clinical trials of patients hospitalized for COVID-19, there was no benefit in terms of disease progression with tocilizumab treatment compared with standard care [18-20]. In 

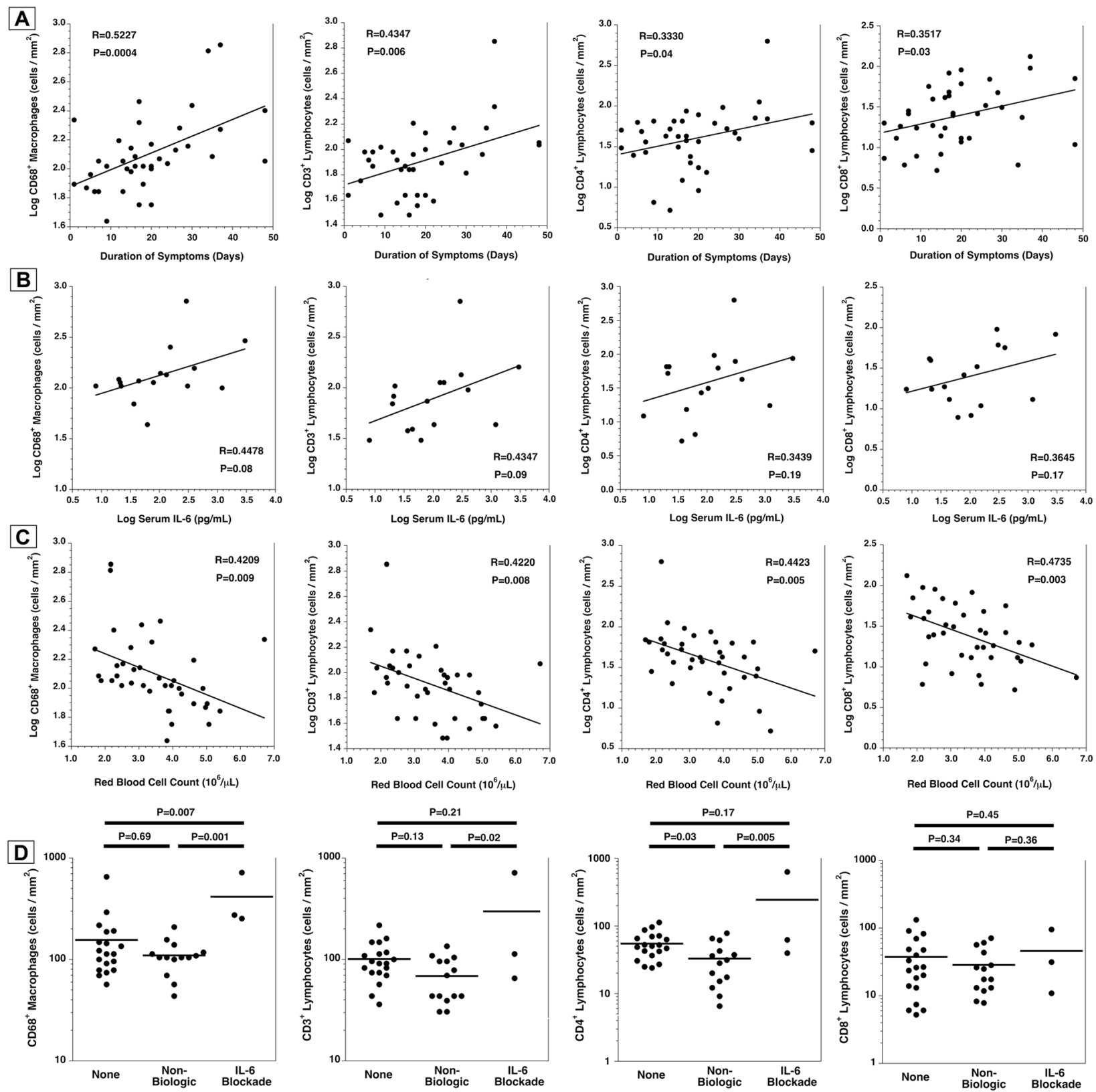

Fig. 6 Relationships between clinical and laboratory features and myocardial inflammatory cell densities. The densities of myocardial $\mathrm{CD} 8^{+}$macrophages, $\mathrm{CD} 3^{+} \mathrm{T}$-lymphocytes, and $\mathrm{CD} 8^{+}$cytotoxic $\mathrm{T}$ lymphocytes were all correlated with the duration of hospitalization, and there was a non-significant correlative association between the density of $\mathrm{CD}^{+}{ }^{+}$helper T-lymphocytes and the duration of symptoms (A). All four inflammatory cell types showed non-significant correlative associations with the serum IL-6 levels (B). All four inflammatory

contrast, recent clinical trials have shown treatment with dexamethasone or hydrocortisone to improve outcomes in critically ill patients with COVID-19 [14-17]. Our observations suggest that part of the efficacy of glucocorticoids in the setting of COVID-19 could be related to reduced cardiac infection by the virus along with reduced myocardial inflammation and myocarditis. cell types were inversely correlated with the serum red blood cell levels (C). The myocardial inflammatory cell densities were also compared between the patients receiving nonbiologic immunosuppression (see Supplementary Table S2), IL-6 blockade without nonbiologic immunosuppression or no immunosuppression (D). Data were analyzed using linear regression $(\mathbf{A}, \mathbf{B}, \mathbf{C})$ or ANOVA with post-test by Bonferroni (D).

In patients with fatal COVID-19 disease, approximately $60-70 \%$ have viremia, with detectable SARS-CoV-2 in the plasma by PCR [3, 4], consistent with our observation that $73 \%$ of the patients had detectable SARS-CoV-2 in the myocardium at autopsy. In addition to the heart, previous studies utilizing RT-PCR on fresh tissue have shown similar levels of SARS-CoV-2 RNA in other organs throughout the 
body $[9,11,13]$. Thus, other organs such as the liver and kidney may also contain a low level of SARS-CoV-2infected cells as was identified in the hearts of some of the patients in this study.

In studies on both critically ill and relatively healthy patients with COVID-19, viremia has been associated with increased disease severity, markers of cytokine storm, and mortality [3, 4, 32, 33]. Nevertheless, few analyses specifically compared viremic and non-viremic patients dying from COVID-19. In one analysis of critically ill patients with COVID-19, no difference in the serum IL-6 levels was noted between patients with or without viremia [4], consistent with our observation on the lack of differences in serum IL-6 levels in the $\mathrm{V}^{+} \mathrm{M}^{-}$and $\mathrm{V}^{-} \mathrm{M}^{-}$groups. In addition, several laboratory values herein suggested that the $\mathrm{V}^{+} \mathrm{M}^{-}$group may experience less cytokine storm than the $\mathrm{V}^{-} \mathrm{M}^{-}$group, including better liver and renal function and lower levels of D-dimer and LDH. Previous studies on SARS-CoV in human and animal models suggested that viral titers diminished with severe disease progression, and that immune dysregulation may not always be closely linked with viremia $[34,35]$. The $\mathrm{V}^{+} \mathrm{M}^{-}$group here may be showing more viremia but less cytokine storm than the $\mathrm{V}^{-}$ $\mathrm{M}^{-}$group.

This single-center study benefits from its systematic approach to detailed histopathologic assessment and use of ISH and NanoString transcriptomic profiling to detect SARS-CoV-2. In our assessment of cardiac SARS-CoV-2 infection, the use of ISH in conjunction with NanoString minimized the effects of peripheral blood contamination due to circulating virions, which may affect other SARSCoV-2 molecular detection methods. However, there are several limitations to this study.

Our study focused only on patients with fatal COVID-19 who underwent autopsy, and these cases represent only a minor fraction of the 183 patients hospitalized with COVID-19 at our institution who died during this time period. In a recent study of the race and ethnicity of the patients dying from COVID-19 during the spring of 2020 in our hospital, $69 \%$ of fatalities were Caucasian patients [36], while Caucasians comprise $56 \%$ of the autopsies in our series. However, this prior study only included deaths within 14 days of presentation, and many of the patients in our study had durations of hospitalization that exceeded 14 days. Also, only a small number of the patients in our study received antiviral therapy (Supplementary Table S2). Thus, the results in this study may not be readily extendible to all patients dying with COVID-19. Likewise, patients who die from COVID-19 tend to be older with more comorbidities than those who survive COVID-19 [37]. In a study of our hospital's SARS-CoV-2 testing facility, the median age of all patients testing positive for SARS-CoV-2 during the spring of 2020 was 47 with $50 \%$ being men [38], compared with a median age of 67 and $66 \%$ men in our autopsy series. Thus, our observations may not be generalizable to all patients with SARS-CoV-2 infection.

This study is also retrospective in nature; the clinical data focused on cardiac function, including biomarker assessment and echocardiography, were limited due to infection control concerns and were driven by clinical assessments in patients whose courses were mostly determined by pulmonary disease and multisystem critical illness. While the viral detection methodologies employed in this study are relatively new, with limited assessment in varying clinical settings, it is unlikely that other common coronaviruses will cause false positive results. The NanoString SARS-CoV-2 target sequences in Supplementary Table S1 were each compared to the other four most common coronaviruses (229E, HKU1, NL63, and OC43) using BLAST, and no significant similarities were found (not shown).

In conclusion, for patients with fatal COVID-19, infection of the heart by SARS-CoV-2 is common but is often limited with only rare infected cells. When present, myocarditis is often a relatively late event in the terminal disease course and associated with need for RRT. Cardiac infection by SARS-CoV-2 is associated with more electrocardiographic changes. Nonbiologic immunosuppression is associated with lower incidences of both myocarditis and cardiac infection by SARS-CoV-2.

\section{Data availability}

Access to primary data not available within the article and supplementary material can be provided to academic researchers with institutional review board approval by the authors upon request to JRS.

Author contributions MB and JRS performed study concept and design. MB, YPH, AJK, CN-C, and JRS performed development of methodology and writing, review and revision of the paper. All authors provided acquisition, analysis, or interpretation of data and read and approved the final paper.

Funding The authors received no specific funding for this work.

\section{Compliance with ethical standards}

Conflict of interest The authors declare no competing interests.

Ethics approval/consent to participate The study was approved by the hospital's human subjects institutional review board (Mass General Brigham IRB Protocol 2002P000527) and was performed in accordance with the Declaration of Helsinki.

Publisher's note Springer Nature remains neutral with regard to jurisdictional claims in published maps and institutional affiliations. 


\section{References}

1. Zhu N, Zhang D, Wang W, Li X, Yang B, Song J, et al. A novel coronavirus from patients with pneumonia in China, 2019. N Engl J Med. 2020;382:727-33.

2. Wiersinga WJ, Rhodes A, Cheng AC, Peacock SJ, Prescott HC. Pathophysiology, transmission, diagnosis, and treatment of coronavirus disease 2019 (COVID-19): a review. JAMA. 2020;324:782-93.

3. Fajnzylber J, Regan J, Coxen K, Corry H, Wong C, Rosenthal A, et al. SARS-CoV-2 viral load is associated with increased disease severity and mortality. Nat Commun. 2020;11:5493.

4. Eberhardt KA, Meyer-Schwickerath C, Heger E, Knops E, Lehmann C, Rybniker J, et al. RNAemia corresponds to disease severity and antibody response in hospitalized COVID-19 patients. Viruses. 2020;12:1045.

5. Lala A, Johnson KW, Januzzi JL, Russak AJ, Paranjpe I, Richter F, et al. Prevalence and impact of myocardial injury in patients hospitalized with COVID-19 infection. J Am Coll Cardiol. 2020;76:533-46.

6. Giustino G, Croft LB, Stefanini GG, Bragato R, Silbiger JJ, Vicenzi $\mathrm{M}$, et al. Characterization of myocardial injury in patients with COVID-19. J Am Coll Cardiol. 2020;76: 2043-55.

7. Basso C, Leone O, Rizzo S, De Gaspari M, van der Wal AC, Aubry MC, et al. Pathological features of COVID-19-associated myocardial injury: a multicentre cardiovascular pathology study. Eur Heart J. 2020;41:3827-35.

8. Escher F, Pietsch H, Aleshcheva G, Bock T, Baumeier C, Elsaesser A, et al. Detection of viral SARS-CoV-2 genomes and histopathological changes in endomyocardial biopsies. ESC Heart Fail. 2020;7:2440-7.

9. Hanley B, Naresh KN, Roufosse C, Nicholson AG, Weir J, Cooke GS, et al. Histopathological findings and viral tropism in UK patients with severe fatal COVID-19: a post-mortem study. Lancet Microbe. 2020;1:e245-53.

10. Bradley BT, Maioli H, Johnston R, Chaudhry I, Fink SL, Xu H, et al. Histopathology and ultrastructural findings of fatal COVID19 infections in Washington State: a case series. Lancet. 2020;396:320-32.

11. Wichmann D, Sperhake JP, Lütgehetmann M, Steurer S, Edler C, Heinemann A, et al. Autopsy findings and venous thromboembolism in patients with COVID-19: a prospective cohort study. Ann Intern Med. 2020;173:268-77.

12. Lindner D, Fitzek A, Bräuninger $\mathrm{H}$, Aleshcheva G, Edler C, Meissner K, et al. Association of cardiac infection with SARSCoV-2 in confirmed COVID-19 autopsy cases. JAMA Cardiol. 2020;5:1281-5.

13. Puelles VG, Lütgehetmann M, Lindenmeyer MT, Sperhake JP, Wong MN, Allweiss L, et al. Multiorgan and renal tropism of SARS-CoV-2. N Engl J Med. 2020;383:590-2.

14. RECOVERY Collaborative Group, Horby P, Lim WS, Emberson JR, Mafham M, Bell JL, et al. Dexamethasone in hospitalized patients with Covid-19-preliminary report. N Engl J Med. 2020; NEJMoa2021436.

15. Tomazini BM, Maia IS, Cavalcanti AB, Berwanger O, Rosa RG, Veiga $\mathrm{VC}$, et al. Effect of dexamethasone on days alive and ventilator-free in patients with moderate or severe acute respiratory distress syndrome and COVID-19: the CoDEX randomized clinical trial. JAMA. 2020;324:1307-16.

16. Angus DC, Derde L, Al-Beidh F, Annane D, Arabi Y, Beane A, et al. Effect of hydrocortisone on mortality and organ support in patients with severe COVID-19: the REMAP-CAP COVID-19 corticosteroid domain randomized clinical trial. JAMA. 2020;324:1317-29.
17. WHO Rapid Evidence Appraisal for COVID-19 Therapies (REACT) Working Group, Sterne JAC, Murthy S, Diaz JV, Slutsky AS, Villar J, et al. Association between administration of systemic corticosteroids and mortality among critically ill patients with COVID-19: a meta-analysis. JAMA. 2020;324:1330-41.

18. Stone JH, Frigault MJ, Serling-Boyd NJ, Fernandes AD, Harvey L, Foulkes AS, et al. Efficacy of tocilizumab in patients sospitalized with Covid-19. N Engl J Med. 2020;383:2333-44.

19. Hermine O, Mariette X, Tharaux PL, Resche-Rigon M, Porcher R, Ravaud $\mathrm{P}$, et al. Effect of tocilizumab vs usual care in adults hospitalized with COVID-19 and moderate or severe pneumonia: a randomized clinical trial. JAMA Intern Med. 2021;181:32-40.

20. Salvarani C, Dolci G, Massari M, Merlo DF, Cavuto S, Savoldi L, et al. Effect of tocilizumab vs standard Care on clinical worsening in patients hospitalized with COVID-19 pneumonia: a randomized clinical trial. JAMA Intern Med. 2021;181:24-31.

21. Kitzman DW, Scholz DG, Hagen PT, Ilstrup DM, Edwards WD. Age-related changes in normal human hearts during the first 10 decades of life. Part II (Maturity): a quantitative anatomic study of 765 specimens from subjects 20 to 99 years old. Mayo Clin Proc. 1988;63:137-46.

22. McCormack CA, Lo Gullo R, Kalra MK, Louissaint A Jr, Stone JR. Reliability of body size measurements obtained at autopsy: impact on the pathologic assessment of the heart. Forensic Sci Med Pathol. 2016;12:139-45.

23. Li Y, Stone JR. The impact of splenectomy on human coronary artery atherosclerosis and vascular macrophage distribution. Cardiovasc Pathol. 2016;25:453-60.

24. Wu H, Larsen CP, Hernandez-Arroyo CF, Mohamed MMB, Caza $\mathrm{T}$, Sharshir M, et al. AKI and collapsing glomerulopathy associated with COVID-19 and APOL1 high-risk genotype. J Am Soc Nephrol. 2020;31:1688-95.

25. Oudit GY, Kassiri Z, Jiang C, Liu PP, Poutanen SM, Penninger, et al. SARS-coronavirus modulation of myocardial ACE2 expression and inflammation in patients with SARS. Eur J Clin Invest. 2009;39:618-25.

26. Champion SN, Stone JR. Immune checkpoint inhibitor associated myocarditis occurs in both high-grade and low-grade forms. Mod Pathol. 2020;33:99-108.

27. Sharma A, Garcia G Jr, Wang Y, Plummer JT, Morizono K, Arumugaswami $\mathrm{V}$, et al. Human iPSC-derived cardiomyocytes are susceptible to SARS-CoV-2 infection. Cell Rep. Med. 2020;1:100052.

28. Jimenez-Guiu $X$, Huici-Sánchez $M$, Romera-Villegas A, Izquierdo-Miranda A, Sancho-Cerro A, Vila-Coll R. Deep vein thrombosis in non-critically ill patients with coronavirus disease 2019 pneumonia: deep vein thrombosis in non-intensive care unit patients. J Vasc Surg Venous Lymphat Disord. 2020;S2213-333X (20)30466-2.

29. Kunutsor SK, Laukkanen JA. Incidence of venous and arterial thromboembolic complications in COVID-19: a systematic review and meta-analysis. Thromb Res. 2020;196:27-30.

30. Colon CM, Barrios JG, Chiles JW, McElwee SK, Russell DW, Maddox WR, et al. Atrial arrhythmias in COVID-19 patients. JACC Clin Electrophysiol. 2020;6:1189-90.

31. Peltzer B, Manocha KK, Ying X, Kirzner J, Ip JE, Thomas G, et al. Outcomes and mortality associated with atrial arrhythmias among patients hospitalized with COVID-19. J Cardiovasc Electrophysiol. 2020;31:3077-85.

32. Hagman K, Hedenstierna M, Gille-Johnson P, Hammas B, Grabbe $\mathrm{M}$, Dillner J, et al. SARS-CoV-2 RNA in serum as predictor of severe outcome in COVID-19: a retrospective cohort study. Clin Infect Dis. 2020; ciaa1285.

33. Chen X, Zhao B, Qu Y, Chen Y, Xiong J, Feng Y, et al. Detectable serum severe acute respiratory syndrome coronavirus 2 viral load (RNAemia) is closely correlated with drastically 
elevated interleukin 6 level in critically ill patients with coronavirus disease 2019. Clin Infect Dis. 2020;71:1937-42.

34. Fehr AR, Perlman S. Coronaviruses: an overview of their replication and pathogenesis. Methods Mol Biol. 2015;1282:1-23.

35. Coperchini F, Chiovato L, Croce L, Magri F, Rotondi M. The cytokine storm in COVID-19: an overview of the involvement of the chemokine/chemokine-receptor system. Cytokine Growth Factor Rev. 2020;53:25-32.

36. Bassett IV, Triant VA, Bunda BA, Selvaggi CA, Shinnick DJ, He W, et al. Massachusetts General Hospital Covid-19 registry reveals two distinct populations of hospitalized patients by race and ethnicity. PLoS One. 2020;15:e0244270.

37. Bae S, Kim SR, Kim MN, Shim WJ, Park SM. Impact of cardiovascular disease and risk factors on fatal outcomes in patients with COVID-19 according to age: a systematic review and metaanalysis. Heart. 2021;107:373-80.

38. Miller TE, Garcia Beltran WF, Bard AZ, Gogakos T, Anahtar $\mathrm{MN}$, Astudillo MG, et al. Clinical sensitivity and interpretation of PCR and serological COVID-19 diagnostics for patients presenting to the hospital. FASEB J. 2020;34:13877-84. 